\title{
WATERBODIES EXTRACTION FROM LANDSAT8-OLI IMAGERY USING A WATER INDEXS-GUIED STOCHASTIC FULLY-CONNECTED CONDITIONAL RANDOM FIELD MODEL AND THE SUPPORT VECTOR MACHINE
}

\author{
Xiaohang Wang ${ }^{1}$, Linlin $\mathrm{Xu}^{1 *}$ \\ ${ }^{1}$ School of Land Science and Technology, China University of Geosciences, Beijing, China \\ wangxiaohang@gmail.com, beyond13031@126.com
}

Commission III, WG III/1

KEY WORDS: Water index, CRF, Landsat, Water extraction, SVM

\begin{abstract}
:
One of the most important applications of remote sensing classification is water extraction. The water index (WI) based on Landsat images is one of the most common ways to distinguish water bodies from other land surface features. But conventional WI methods take into account spectral information only form a limited number of bands, and therefore the accuracy of those WI methods may be constrained in some areas which are covered with snow/ice, clouds, etc. An accurate and robust water extraction method is the key to the study at present. The support vector machine (SVM) using all bands spectral information can reduce for these classification error to some extent. Nevertheless, SVM which barely considers spatial information is relatively sensitive to noise in local regions. Conditional random field (CRF) which considers both spatial information and spectral information has proven to be able to compensate for these limitations. Hence, in this paper, we develop a systematic water extraction method by taking advantage of the complementarity between the SVM and a water index-guided stochastic fully-connected conditional random field (SVM-WIGSFCRF) to address the above issues. In addition, we comprehensively evaluate the reliability and accuracy of the proposed method using Landsat- 8 operational land imager (OLI) images of one test site. We assess the method's performance by calculating the following accuracy metrics: Omission Errors (OE) and Commission Errors (CE); Kappa coefficient (KP) and Total Error (TE). Experimental results show that the new method can improve target detection accuracy under complex and changeable environments.
\end{abstract}

\section{INTRODUCTION}

Terrestrial surface water bodies are among the most vital earth resources for human. However, they are vulnerable to globalscale impacts from land use/cover changes (LUCC), climate changes, and other forms of environmental change in the world (Alderman et al., 2012; Huntington et al., 2006; Murray et al., 2012; Sun et al., 2012; Vörösmarty et al., 2000). Changes in terrestrial surface water bodies may result in continuous but unexpected variations from socioeconomic development to ecological environment. Thus, comprehensive and accurate mapping of water bodies to describe its spatial and temporal distribution changes is of crucial importance for both academic research and related policy-making (Morss et al., 2005).

With the rapid development of remote sensing technology, remote sensing has become an important source of information for data analysis and processing, especially in the surface water research. Satellite sensors with different spatial and temporal resolution have been successfully applied to analyze and monitor water bodies. Landsat satellites have been the most widely used optical sensors for surface water monitoring among various satellite sensors due to its noticeable data availability and short revisit interval (Cohen et al., 2004; Van Dijk et al., 2010).

Many methods have been proposed to extract water bodies, which can be categorized into five basic categories: (a) spectral unmixing (De Asis et al. 2008; Rogers et al. 2004; Sethre et al., 2005); (b) single-band thresholding (Jain et al. 2005; Klein et al. 2014), which applies an optimized threshold to extract water from a single spectral band information and is frequently affected by heterogeneous false signals (Verpoorter et al., 2012); (c) supervised or unsupervised classification (Lu et al., 2007; Otukei et al., 2010; Sun et al., 2014; Tulbure et al., 2013), which are the commonly used algorithms in remote sensing, such as the support vector machine (SVM) and the K-Means algorithm. The former heavily relies on a priori expertise to choose suitable training samples, while the latter is easily affected by environment noise; (d) water indexes (WIs) (McFeeters et al., 1996; Feyisa et al., 2014; Xu, 2006), in which band math of two or more bands are used to enhance the discrepancy between water bodies and land surface features; (e) combinations of various methods (Jiang et al., 2012; Sheng et al., 2008; Sun et al., 2012; Verpoorter et al., 2012; Yang et al., 2015), such as the modified fuzzy clustering method based on WIs (WIMFCM).

Among the above methods, WIs are the most popular approach for water extraction (Fisher et al., 2013). They are designed to highlight the water pixels through enhancing the separability between water bodies and nonwater while suppressing environmental noise. Commonly effective and widely used WIs were listed in Table 1, based on the Landsat8 OLI imagery.

The Normalized Difference Water Index (NDWI) proposed by McFeeters (1996) is one of the most used WIs (McFeeters et al., 1996). However, $\mathrm{Xu}$ (2006) found that it is difficult to discriminate built-up land from water pixels when using the NWDI. In order to overcome the deficiency, he formulated the modified normalized-difference water index (MNDWI) that improves the separability of water and urbanized area (Xu,2006). The aforementioned WIs are usually inefficient in areas that include shadow and dark surfaces. Based on this issue, Feyisa 


\begin{tabular}{l|l|l}
\hline \multicolumn{1}{l|}{ Index } & \multicolumn{1}{c}{ Formula } & Reference \\
\hline NDWI & $\left(\rho_{\text {Green }}-\rho_{\text {NIR }}\right) /\left(\rho_{\text {Green }}+\rho_{\text {NIR }}\right)$ & McFeeters 1996 \\
MNDWI & $\left(\rho_{\text {Green }}-\rho_{\text {SWIR } 1}\right) /\left(\rho_{\text {Green }}+\rho_{\text {SWIR } 1}\right)$ & Xu 2005 \\
AWEI & $4 \times\left(\rho_{\text {Green }}-\rho_{\text {SWIR1 }}\right)-\left(0.25 \times \rho_{\text {NIR }}+2.75 \times \rho_{\text {SWIR2 }}\right)$ & Feyisa et al.2014 \\
AWEI $_{\text {sh }}$ & $\rho_{\text {Blue }}+2.5 \times \rho_{\text {Green }}-1.5 \times\left(\rho_{\text {NIR }}+\rho_{\text {SWIR1 }}\right)-0.25 \times \rho_{\text {SWIR2 }}$ & Feyisa et al.2014 \\
& & \\
\hline
\end{tabular}

Table1. Common water indices used for the extraction of water features ( $\rho$ : the reflectance value of spectral bands)

(2014) formulates a new method, called Automated water extraction index (AWEI) (Feyisa et al., 2014).

Despite their excellent performance with respect to specific noise, WIs still remain many problems to be solved. For instance, when using WIs, water pixels with variable chemical and physical components may show a lower value than pure water pixels. The reason is that they only use partial spectral information to discriminate water bodies from other surfaces. In addition, the performance of WIs should be comprehensively tested prior to application which is rarely mentioned in the previous literature as far as we know (Campos et al., 2012). Secondly, WIs are generally intended for prescriptive conditions as mention above. Their performance therefore suffers from insufficiency in snowcovered areas where land surface pixels with snow can also show a high value. The main reason is that they have not taken the background information into consideration (Ji et al., 2015).

SVM can use all of the bands information to extract water bodies, but it relies on a priori expertise and discards the spatial correlation, which may be sensitive to noise in different environment. Conditional random field (CRF) has the intrinsic ability to incorporate contextual information in both the labels and the observed data. Meanwhile, the arbitrary function of observed features can be incorporated into their training process (Cao et al., 2016). Focuses on improving accuracy and robustness, reducing useless spectrum information, we develop a systematic water extraction method named SVM-WI guided stochastic fully-connected CRF (SVM-WIGSFCRF) method.

\section{METHODOLOGY}

In this section, the overall workflow is divided into five main parts (Figure 1): (1) data collection; (2) image pre-processing, including radiometric calibration, atmospheric correction and image cropping; (3) water extraction by the related methods; (4) SFCRC segmentation, using the preliminary probabilistic graph of SVM and the generated images by WIs to get an ideal classification result; (5) result analysis.

\subsection{Study area and feature extraction}

In order to test the proposed method of this study, the Landsat 8 OLI images are acquired from the United States Geological Survey (USGS) portal (http://earthexplorer.usgs.gov/). The study area information is shown in the Figure 2.

Radiometric calibration and atmospheric correction of the satellite imagery are prerequisite for generating consistent and high-quality image materials (Chander et al., 2009). WIs are used to generate new gray images and we choose the best result of them as the basic gray image data for the SFCRF model to segment and infer. In addition, gray values of the WI image are linearly normalized into $[0,1]$.

\subsection{Stochastic fully-connected conditional random field}

The conventional CRF considering local spatial information along with spectral information has a proven capability to classify Landsat images, therefore, CRFs have been widely used in the remote sensing (Salmon et al., 2015; Li et al., 2015; Xu et al., 2017). The fully-connected CRF (FCRF) addresses the correlation effect in the global image scale is better than the conventional CRF that considers the correlation effect in a local area (Krähenbühl et al., 2011).

The stochastic FCRF (SFCRF) model is proposed to maintain the advantage of FCRF but reduce its computational cost by using a stochastic clique approach (Shafiee et al., 2014; Xu et al., 2015; $\mathrm{Xu}$ et al., 2016). SVM-WIGSFCRF is a mixed model, where probabilistic graph generated by SVM and WI are used as the guide to extract water bodies, while the stochastic clique is used to determine the connectivity among nodes in a fully-connected graph.

We assume that $\mathrm{x}_{i}$ and $\mathrm{y}_{i}$ denote respectively the pixel value and the class label of a site in the WIs image. $N$ is the total number of pixels in the image. The Landsat OLI image can be expressed as $X=\left\{x_{i} \mid i=1,2, \cdots, N\right\}$ and $Y=\left\{y_{i} \mid i=1,2, \cdots, N\right\}$. Thus, water bodies extraction aims to infer $Y$ given $X$ by maximizing the following conditional probability distribution:

$P(Y \mid X)=\frac{1}{Z(X)} \exp \left\{-\sum_{i} \psi_{u}\left(y_{i}, X\right)-\sum_{(i, j) \in C} \psi_{p}\left(y_{i}, y_{j}, X\right)\right\}(1)$

where the function $Z(X)$ is the partition function in order to ensure that the distribution sums to unity. The unary potential $\sum_{i} \psi_{u}\left(y_{i}, X\right)$ as a posterior probability of the class labels given the observation data, disregarding the interaction between itself and other pixels in the image. The $\sum_{(i, j) \in C} \psi_{p}\left(y_{i}, y_{j}, X\right)$ usually named as the pairwise potential, which means the relationship between current observed data, its neighboring observed data and their corresponding labels. In addition, $\mathrm{C}$ is defined as the set of clique structure in the random field which determines the connectivity among nodes in the neighborhood. 
The International Archives of the Photogrammetry, Remote Sensing and Spatial Information Sciences, Volume XLII-3, 2018 ISPRS TC III Mid-term Symposium “Developments, Technologies and Applications in Remote Sensing”, 7-10 May, Beijing, China

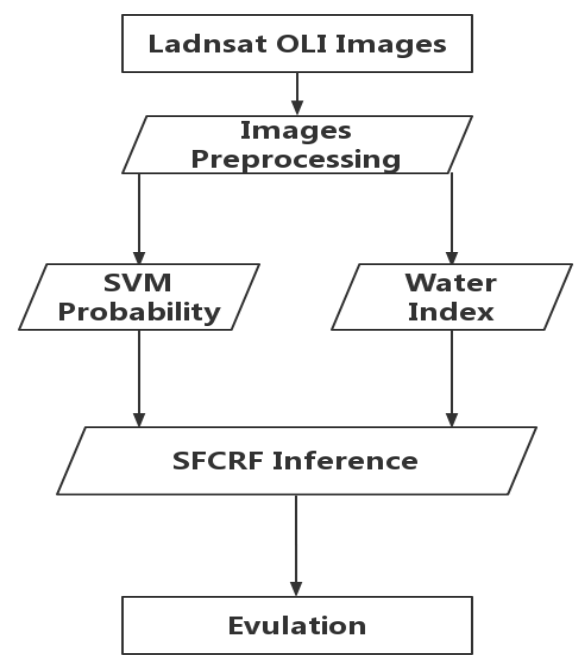

Figure 1. Flowchart of SVM-WIGSFCRF algorithm for water extraction

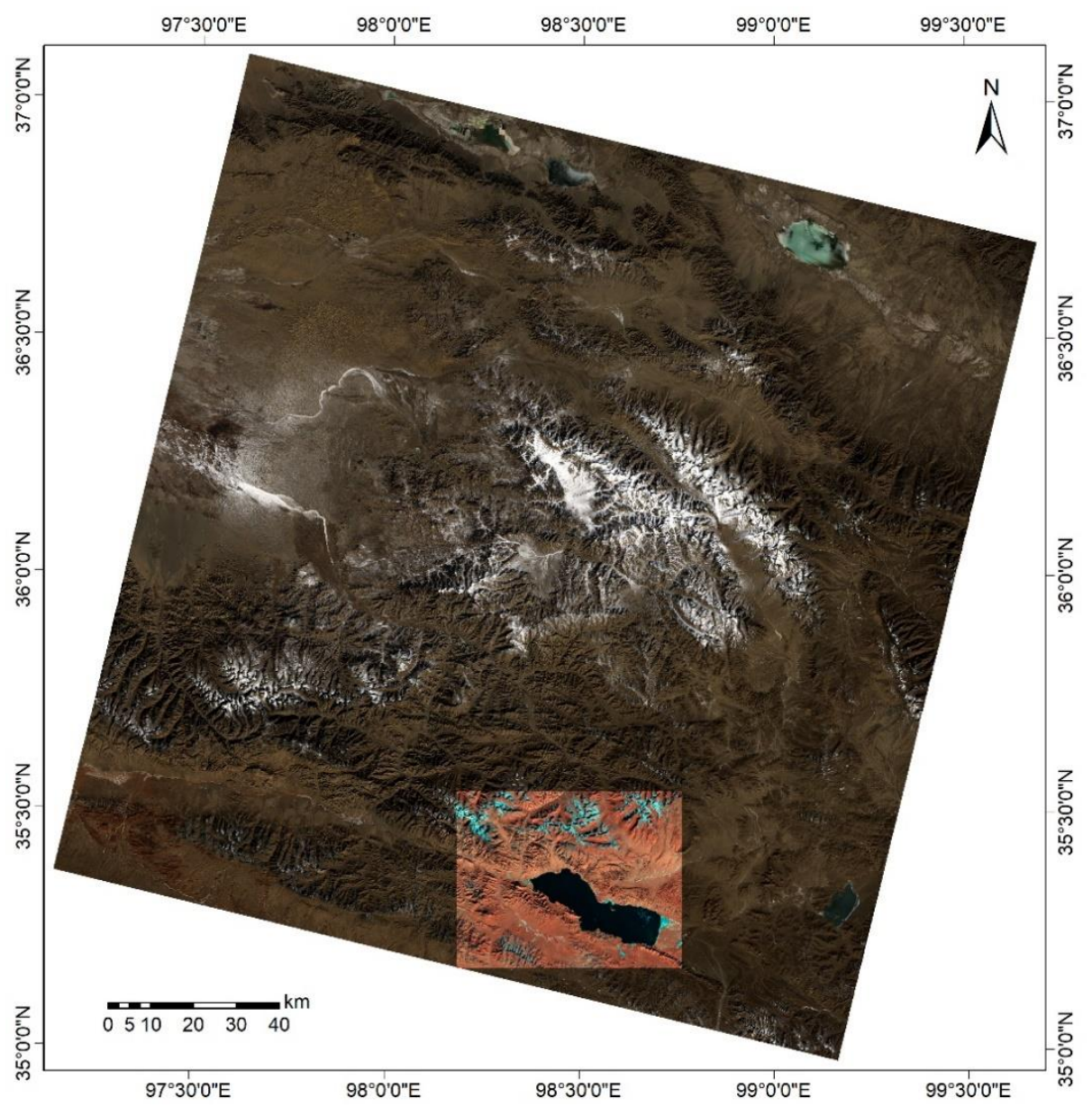

Figure 2. Study area: Donggei Cuona Lake (Path/ROW 134/35 2016/12/11)

\subsection{Unary potential}

The unary potential $\sum_{i} \psi_{u}\left(y_{i}, X\right)$ is usually computed using a discriminative classifier to infer the initial probabilistic graph of the image (Cao et al., 2016; Salmon et al., 2015; Wang et al., 2016). The unary potential function is formulated as below:

$$
\psi_{u}\left(y_{i}, X\right)=-\log (p(y i \mid x i)
$$

where $p(y i \mid x i)$ is the posterior probability of $y i$ given $x i$ based on the library for SVM (LIBSVM). In order to test the practicability of the proposed method and reduce the processing time of SVM, a small number of samples are chosen. Water pixels (1656) and other land surface pixels (2173) are chosen as the train data. 


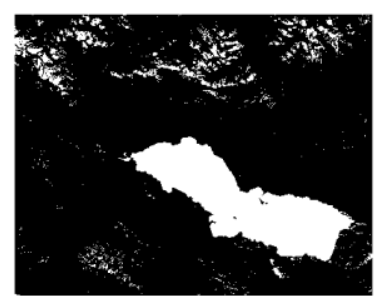

AWEInsh

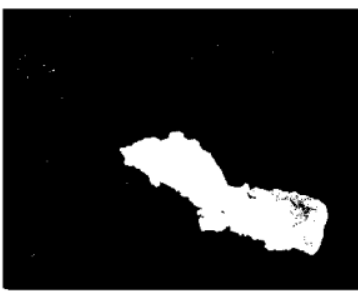

SVM

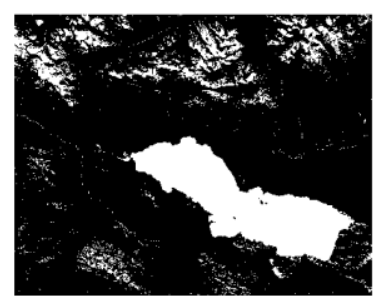

MNDWI

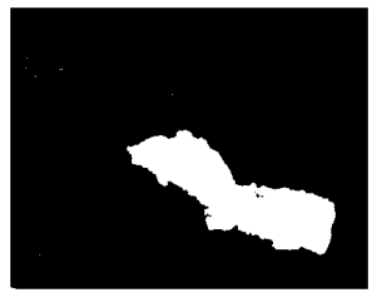

SVM-WIGCRF

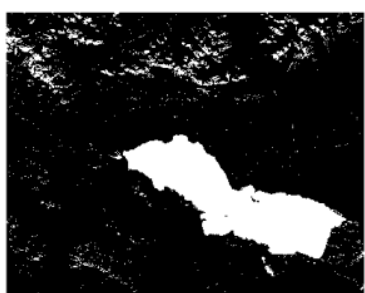

NDWI

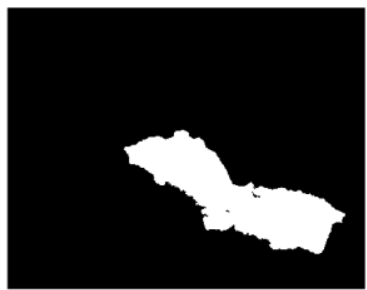

Ground Truth

Figure 3. Water extraction results achieved by different methods. Traditional WI methods are sensitive to ice/snow lead to a lower accuracy than other methods. SVM performs better than WIs, but is still affected by ice/snow and yields many false alarms. SVM-

WIGCRF achieve the best result and produces a clean background.

\begin{tabular}{|c|c|c|c|c|}
\hline Method & CE (\%) & OE (\%) & KP (\%) & TE (\%) \\
\hline NDWI & 27.39 & 0.21 & 81.26 & 27.60 \\
\hline MNDWI & 40.76 & 0.11 & 68.68 & 40.87 \\
\hline AWEInsh & 24.97 & 0.14 & 83.28 & 25.11 \\
\hline SVM & 0.25 & 4.55 & 97.38 & 4.80 \\
\hline SVM-WIGSCRF & 1.30 & 1.90 & 98.21 & 3.20 \\
\hline
\end{tabular}

Table 2. Summary of accuracies for the proposed method over the study area

\subsection{Pairwise potential}

To incorporate the spatial information, the pairwise potential can be expressed as follows:

$$
\psi_{p}\left(y_{i}, y_{j}, X\right)=-\lambda \cdot \mu\left(y_{i}, y_{j}\right) \cdot P_{i j}
$$

where $\mu\left(y_{i}, y_{j}\right)$ is implemented according to the Potts model and $\lambda$ is a trade-off coefficient between the two potential terms. As we method before in (1), $i$ and $j$ must be included in the clique $C$. Accordingly, the widely used pairwise clique structure is adopted here:

$$
\begin{gathered}
C=\{C(i)\} \\
C(i)=\left\{(i, j) \mid j \in N_{i}, I(i, j)=1\right\}
\end{gathered}
$$

$N_{i}$ usually represents the whole neighborhood of node $i$ in the FCCRF model, which is expressed as below:

$$
N_{i}=\{j \mid j=1: N, j \neq i\}
$$

$I\left(i_{J} j\right)$ is a clique indicator function determining whether two nodes can be connected, according to a stochastic measure:

$$
I\left(i_{J} j\right)=\left\{\begin{array}{lr}
1 & \text { if } \gamma \cdot P_{i j} Q_{i j} \geq \varphi \\
0 & \text { othersiwse }
\end{array}\right.
$$

Symbol $P_{i j}$ and $Q_{i j}$ denote respectively the data similarity likelihood and the probabilistic spatial closeness measurement. The data similarity likelihood $P_{i j}$ is expressed as (Deledalle et al., 2009):

$$
P_{i j}=4 L\left(\frac{a_{i} a_{j}}{a_{i}^{2}+a_{j}^{2}}\right)
$$

Where $L$ reveals the complexity of the image. The amplitude values are expressed by $a_{i}=\sqrt{x_{i}}$ and $a_{j}=\sqrt{x_{j}}$.The probabilistic spatial closeness measurement between pixel and is defined as below:

$$
Q_{i j}=\exp \left(-\frac{\left(L_{i r}-L_{i j}\right)^{2}+\left(L_{i c}-L_{i c}\right)^{2}}{2 \sigma^{2}}\right)
$$

where $L_{i r}$ and $L_{i j}$ are respectively the row and column locations of site $i$ in image space, and $\sigma$ determines the spatial scale. Based on the Potts model, $\mu\left(y_{i}, y_{j}\right)$ in (3) can be expressed as follows:

$$
\mu\left(y_{i}, y_{j}\right)=\left\{\begin{array}{rr}
1 & y_{i} \neq y_{j} \\
0 & \text { otherwise }
\end{array}\right.
$$

In summary, the binary classification of OLI image is achieved by using the above described unary and pairwise potentials. Water bodies is successfully extracted from land surfaces according to the maximum a posterior (MAP), such that

$$
Y^{*}=\underset{\hat{Y}}{\operatorname{argmax}} P(Y \mid X)
$$

where $Y^{*}$ is the best label configuration in the set $Y$ that maximizes $P(Y \mid X)$. In order to find $Y^{*}$, the energy function $\sum_{i} \psi_{u}\left(y_{i}, X\right)+\sum_{(i, j) \in C} \psi_{p}\left(y_{i}, y_{j}, X\right)$ of (1) is minimized by graph-cut approach (Kohli et al., 2007). 


\subsection{Experiment results}

For this study, the "true" water bodies are refined by means of associated high-resolution Google Earth ${ }^{\mathrm{TM}}$ imagery. Ground truth, and experiment results with the different methods are shown in Figure 3. The results regarding the numerical evaluation were reported in Table 2 . We choose the 0 as the threshold value of WIs. We can find that WIs may have a higher error than other methods, when they choose the 0 -theoretical threshold. Therefore, we must change threshold value varying with the environment. SVM and SVM-WIGCRF can be adapted in a complicated environment and the latter have the better accuracy. As we can see from the Table2, SVM-WIGCRF obtains the best result in three indices, which illustrates the effectiveness and stability of the proposed model.

\section{CONCLUSIONS}

In this paper, we presented a SVM-WIGSFCRF algorithm for the purpose of water extraction. The proposed method comparing the traditional methods is more capable of modelling large-scale spatial correlation effect by the use of stochastic clique approach, and thereby is more tailored to the changeable water environment The experiments conducted on the large-scale image demonstrate that SVM-WIGSFCRF can better delineate water bodies, without being significantly affected by background and target heterogeneities. Further experiments in different regions and seasons are still necessary to enhance the robustness of the method given the high diversity of water bodies globally.

\section{ACKNOWLEDGEMENTS}

This work was supported by the National Natural Science Foundation of China Grant 41501410.

\section{REFERENCES}

Alderman, K., Turner, L. R., \& Tong, S. (2012). Floods and human health: a systematic review. Environment international, 47, 37-47.

Campos, J. C., Sillero, N., \& Brito, J. C. (2012). Normalized difference water indexes have dissimilar performances in detecting seasonal and permanent water in the Sahara-Sahel transition zone. Journal of Hydrology, 464, 438-446.

Cao, G., Zhou, L., \& Li, Y. (2016). A new change-detection method in high-resolution remote sensing images based on a conditional random field model. International Journal of Remote Sensing, 37(5), 1173-1189.

Chander, G., Markham, B. L., \& Helder, D. L. (2009). Summary of current radiometric calibration coefficients for Landsat MSS, TM, ETM+, and EO-1 ALI sensors. Remote sensing of environment, 113(5), 893-903.

Cohen, W. B., \& Goward, S. N. (2004). Landsat's role in ecological applications of remote sensing. AIBS Bulletin, 54(6), 535-545.

De Asis, A. M., Omasa, K., Oki, K., \& Shimizu, Y. (2008). Accuracy and applicability of linear spectral unmixing in delineating potential erosion areas in tropical watersheds. International Journal of Remote Sensing, 29(14), 4151-4171.

Feyisa, G. L., Meilby, H., Fensholt, R., \& Proud, S. R. (2014).
Automated Water Extraction Index: A new technique for surface water mapping using Landsat imagery. Remote Sensing of Environment, 140, 23-35.

Fisher, A., \& Danaher, T. (2013). A water index for SPOT5 HRG satellite imagery, New South Wales, Australia, determined by linear discriminant analysis. Remote Sensing, 5(11), 5907-5925.

Huntington, T. G. (2006). Evidence for intensification of the global water cycle: review and synthesis. Journal of Hydrology, 319(1), 83-95.

Jain, S. K., Singh, R. D., Jain, M. K., \& Lohani, A. K. (2005). Delineation of flood-prone areas using remote sensing techniques. Water Resources Management, 19(4), 333-347.

Ji L, Geng X, Sun K, et al. Target detection method for water mapping using Landsat 8 OLI/TIRS imagery[J]. Water, 2015, 7(2): 794-817.

Jiang, Z., Qi, J., Su, S., Zhang, Z., \& Wu, J. (2012). Water body delineation using index composition and HIS transformation. International journal of remote sensing, 33(11), $3402-3421$

Klein, I., Dietz, A. J., Gessner, U., Galayeva, A., Myrzakhmetov, A., \& Kuenzer, C. (2014). Evaluation of seasonal water body extents in Central Asia over the past 27 years derived from medium-resolution remote sensing data. International Journal of Applied Earth Observation and Geoinformation, 26, 335-349.

Kohli, P., \& Torr, P. H. (2007). Dynamic graph cuts for efficient inference in markov random fields. IEEE transactions on pattern analysis and machine intelligence, 29(12), 2079-2088.

Krähenbühl, P., \& Koltun, V. (2011). Efficient inference in fully connected crfs with gaussian edge potentials. In Advances in neural information processing systems (pp. 109-117).

Li, F., Xu, L., Siva, P., Wong, A., \& Clausi, D. A. (2015). Hyperspectral image classification with limited labeled training samples using enhanced ensemble learning and conditional random fields. IEEE Journal of Selected Topics in Applied Earth Observations and Remote Sensing, 8(6), 2427-2438.

Lu, D., \& Weng, Q. (2007). A survey of image classification methods and techniques for improving classification performance. International journal of Remote sensing, 28(5), 823-870.

Morss, R. E., Wilhelmi, O. V., Downton, M. W., \& Gruntfest, E. (2005). Flood risk, uncertainty, and scientific information for decision making: lessons from an interdisciplinary project. Bulletin of the American Meteorological Society, 86(11), 1593-1601.

McFeeters, S. K. (1996). The use of the Normalized Difference Water Index (NDWI) in the delineation of open water features. International journal of remote sensing, 17(7), 14251432.

Murray, N. J., Phinn, S. R., Clemens, R. S., Roelfsema, C. M., \& Fuller, R. A. (2012). Continental scale mapping of tidal flats across East Asia using the Landsat archive. Remote Sensing, 4(11), 3417-3426.

Otukei, J. R., \& Blaschke, T. (2010). Land cover change 
assessment using decision trees, support vector machines and maximum likelihood classification algorithms. International Journal of Applied Earth Observation and Geoinformation, 12, S27-S31.

Rogers, A. S., \& Kearney, M. S. (2004). Reducing signature variability in unmixing coastal marsh Thematic Mapper scenes using spectral indices. International Journal of Remote Sensing, 25(12), 2317-2335.

Salmon, B. P., Kleynhans, W., Olivier, J. C., Schwegmann, C. P., \& Olding, W. C. (2015, July). A multi-tier higher order conditional random field for land cover classification of multitemporal multi-spectral landsat imagery. In Geoscience and Remote Sensing Symposium (IGARSS), 2015 IEEE International (pp. 4372-4375). IEEE.

Sethre, P. R., Rundquist, B. C., \& Todhunter, P. E. (2005). Remote detection of prairie pothole ponds in the Devils Lake Basin, North Dakota. GIScience \& Remote Sensing, 42(4), 277296.

Shafiee, M. J., Wong, A., Siva, P., \& Fieguth, P. (2014, October). Efficient bayesian inference using fully connected conditional random fields with stochastic cliques. In Image Processing (ICIP), 2014 IEEE International Conference on (pp. 4289-4293). IEEE.

Sheng, Y., Shah, C. A., \& Smith, L. C. (2008). Automated image registration for hydrologic change detection in the lake-rich Arctic. IEEE geoscience and remote sensing letters, 5(3), 414418.

Sun, F., Sun, W., Chen, J., \& Gong, P. (2012). Comparison and improvement of methods for identifying waterbodies in remotely sensed imagery. International journal of remote sensing, 33(21), 6854-6875.

Sun, F., Zhao, Y., Gong, P., Ma, R., \& Dai, Y. (2014). Monitoring dynamic changes of global land cover types: fluctuations of major lakes in China every 8 days during 2000 2010. Chinese Science Bulletin, 59(2), 171-189.

Tulbure, M. G., \& Broich, M. (2013). Spatiotemporal dynamic of surface water bodies using Landsat time-series data from 1999 to 2011. ISPRS Journal of Photogrammetry and Remote Sensing, 79, 44-52.

Van Dijk, A. I. J. M., \& Renzullo, L. J. (2011). Water resource monitoring systems and the role of satellite observations. Hydrology and Earth System Sciences, 15(1), 39.

Verpoorter, C., Kutser, T., \& Tranvik, L. (2012). Automated mapping of water bodies using Landsat multispectral data. Limnology and Oceanography: Methods, 10(12), 10371050 .

Vörösmarty, C. J., Green, P., Salisbury, J., \& Lammers, R. B. (2000). Global water resources: vulnerability from climate change and population growth. science, 289(5477), 284-288.

Wang, H., Wang, C., \& Wu, H. (2016). Using GF-2 imagery and the conditional random field model for urban forest cover mapping. Remote Sensing Letters, 7(4), 378-387.

$\mathrm{Xu}, \mathrm{H}$. (2006). Modification of normalised difference water index (NDWI) to enhance open water features in remotely sensed imagery. International journal of remote sensing, 27(14), 30253033.

Xu, L., Javad Shafiee, M., Wong, A., Li, F., Wang, L., \& Clausi, D. (2015). Oil spill candidate detection from SAR imagery using a thresholding-guided stochastic fully-connected conditional random field model. In Proceedings of the IEEE Conference on Computer Vision and Pattern Recognition Workshops (pp. 7986).

Xu, L., Shafiee, M. J., Wong, A., \& Clausi, D. A. (2016). Fully Connected Continuous Conditional Random Field With Stochastic Cliques for Dark-Spot Detection In SAR Imagery. IEEE Journal of Selected Topics in Applied Earth Observations and Remote Sensing, 9(7), 2882-2890.

Xu, L., Clausi, D. A., Li, F., \& Wong, A. (2017). Weakly Supervised Classification of Remotely Sensed Imagery Using Label Constraint and Edge Penalty. IEEE Transactions on Geoscience and Remote Sensing, 55(3), 1424-1436.

Yang, Y., Liu, Y., Zhou, M., Zhang, S., Zhan, W., Sun, C., \& Duan, Y. (2015). Landsat 8 OLI image based terrestrial water extraction from heterogeneous backgrounds using a reflectance homogenization approach. Remote Sensing of Environment, 171, 14-32. 